\title{
Patient and Public Preferences for Coordinated Care in Switzerland: Development of a Discrete Choice Experiment
}

\author{
Anna Nicolet ${ }^{1}\left[\right.$. Clémence Perraudin ${ }^{1} \cdot$ Joël Wagner $^{2} \cdot$ Ingrid Gilles $^{1} \cdot$ Nicolas Krucien $^{3}$. \\ Isabelle Peytremann-Bridevaux ${ }^{1} \cdot$ Joachim Marti $^{1}$
}

Accepted: 12 December 2021 / Published online: 24 January 2022

(c) The Author(s) 2022

\begin{abstract}
Objective Our objective was to develop and test a discrete choice experiment (DCE) eliciting public and patient preferences for better-coordinated care in Switzerland.

Methods We applied a multistage mixed-methods procedure using qualitative and quantitative approaches. First, to identify attributes, we performed a review of the DCE literature in healthcare with a focus on chronic care. Next, attribute selection involved stakeholders $(N=7)$ from various healthcare sectors to select the most relevant and actionable attributes, followed by three organized focus groups involving the general public and patients $(N=21)$ to verify the selection and the clarity of the DCE tasks and explanations. Finally, we conducted an online pilot in the target population to test the survey and obtain priors for a final six tested attributes to refine the final design of the experiment.

Results After identifying an initial 33 attributes, a final list of six attributes was selected following stakeholder involvement and the three focus groups involving the target population. At the online pilot-testing stage with 301 participants, the majority of respondents found the DCE choice tasks socially relevant for Switzerland but challenging. The quality of the answers was relatively high. Most attributes had signs matching those in the literature and focus group discussions.

Conclusion This article will be useful to researchers designing DCEs from a broad health policy perspective. The multistage approach involving a range of stakeholders was essential for the development of a DCE that is relevant for policy makers and well-accepted by the general public and patients.
\end{abstract}

\section{Introduction}

The increasing number of chronic and multimorbid patients poses considerable challenges to the organization and financing of health systems worldwide. These patients have complex needs and transition frequently between care settings [1], requiring specific strategies for efficient healthcare delivery. Such strategies cover several dimensions of care organization (e.g., provider choice,

Anna Nicolet

Anna.Nicolet@unisante.ch

1 Center for Primary Care and Public Health (Unisanté), University of Lausanne, Biopôle 2 SV-A, Route de la Corniche 10, 1010 Lausanne, Switzerland

2 Department of Actuarial Science, Faculty of Business and Economics (HEC), and Swiss Finance Institute, University of Lausanne, Chamberonne -

Extranef, 1015 Lausanne, Switzerland

3 Patient-Centered Research, Evidera, London, UK gatekeeping, data sharing, professional coordination), with the overarching aim of facilitating better-coordinated integrated care while accounting for the needs and preferences of individuals [2-6].

In Switzerland, the health system is highly complex and fragmented [7]; it also has a relatively strong focus on acute and specialist care, with healthcare expenditures dedicated to primary care among the lowest across countries within the Organisation for Economic Co-operation and Development [8]. The division of responsibilities and the specific provider payment and financing mechanisms make chronic care integration particularly challenging in this country [9]. Additional challenges for the Swiss health system include the high proportion of gross domestic product spent on healthcare $(>12 \%)$ and the high level of contributions from households, including insurance premiums and out-of-pocket payments [10]. Better care integration can eliminate inefficiencies in the system through enhanced coordination and better alignment with patient needs $[9,11,12]$. In this context, research is needed on the 


\section{Key Points}

We demonstrate the application of a multistage, mixedmethods process with a range of stakeholders to develop a discrete choice experiment (DCE) survey, ensuring that the final DCE design was both appropriate and actionable from the perspective of the primary stakeholders and understandable and relevant for patients and the public.

Various stages provided important feedback on the attributes related to care coordination and set more focus on informal care and financial barriers for chronically ill patients.

Our study may be useful for future researchers planning to apply mixed-methods approach for the development of DCEs in a broad policy-relevant context.

acceptability of and incentives for better-coordinated care models in Switzerland [13].

Other studies have suggested that care coordination initiatives (e.g., in emergency department use, palliative care, mental health, or in patients with cancer) are effective, lead to favorable outcomes, and reduce healthcare costs [14-17]. Although the need for new models of care targeting coordination is recognized, there remains no consensus on the exact definition, tasks, and executors of care coordination, which makes implementation even more challenging $[9,18]$. It can be illustrated by the diversity of characteristics used to describe the concept of coordinated or chronic care in the DCE studies: case management, multidisciplinary care, shared decision making, the range of activities provided by care coordinators, and the responsibility for the coordination [19-21]. For Switzerland, the complexity also lies in high decentralization, weakened primary care, payment mechanisms for providers lacking incentives for coordination activities, and non-standardized use of electronic patient records $[9,17,22,23]$, elements also highlighted by the only DCE study on financing of coordinated care in Switzerland [24].

To ensure policies are accepted and adopted, and to maximize the value of healthcare, it is crucial that the preferences of the general public and patients are taken into account $[25,26]$. This can also allow decision makers to prioritize the most important changes for implementation $[27,28]$. For example, implementing general recommendations following a "one size fits all" approach could be inefficient, since patients value chronic care delivery and opportunities for its improvement in different ways $[19,29-31]$.

To quantify public preferences and trade-offs for care access and delivery characteristics, the discrete choice experiment (DCE) approach is widely used in studies of individual and social preferences in health and healthcare [32-35], including studies focused on healthcare delivery for patients [19, 36-40]. The method involves asking participants to indicate their preferred hypothetical scenarios, and the preferences are derived after a series of such choices are made. DCEs have been used in broad healthcare contexts in numerous countries (e.g., the UK [41-44], the USA $[45,46]$, the Netherlands [47-50], Australia [51, 52], and Canada [53]) and in countries reaching for major healthcare improvements, such as Malawi [54], Cambodia [55], Ethiopia [56], Tanzania [57], and Zambia [58]. However, studies using DCEs are often criticized for omitting or not providing enough detail on the process of the survey development and, specifically, the selection of the attributes and their levels [59-61]. Therefore, best practice now encourages detailed reporting of the process of DCE development to ensure transparency of the process and provide guidance for future studies to enable more standardized documentation practices [32, 61-63]. Earlier reviews of DCEs found numerous approaches to the development of DCEs, with the two most frequent being the qualitative approach and mixed methods [54, 64-68], and the latter proving to be less time and resource consuming [69].

In the Swiss context, earlier studies [24, 70] used DCEs to quantify the trade-offs of the Swiss public and service providers between conventional healthcare insurance plans and their possible modifications. However, in the broader health policy perspective, with many existing initiatives addressing issues of cost containment, preferences of the Swiss public for potential ways of optimizing healthcare delivery have not been extensively studied.

In this article, we describe the development of a DCE aimed at eliciting preferences from Swiss residents aged $\geq 50$ years for new ways of accessing and delivering care in Switzerland. We focused on the population aged $\geq 50$ years as such individuals are more likely to benefit from new coordinated care models because of the onset of multiple chronic diseases, with statistics showing that $22 \%$ of this population [71] have multimorbidity and associated increasing healthcare costs. We followed recommendations from recent reviews that highlighted the need for thorough descriptions of DCE development [69].

\section{Methods}

\subsection{Attribute Identification}

The initial step of our DCE development was to identify a list of the relevant attributes describing features of healthcare delivery, organization, and financing relevant to care coordination in Switzerland. Importantly, the potential 
attributes needed to be relevant to patients with chronic disease and the general population and reflect their needs for healthcare delivery in Switzerland. They also needed to be measurable, actionable, and realistic for policy implementation to avoid subjective or personalized attributes. The attributes did not necessarily need to fit the current regulatory framework and could therefore be innovative.

This initial list of attributes was identified from a targeted review of the international scientific literature as well as from the research team's expertise (medical and health systems) in the Swiss context. Using published systematic reviews of DCEs in health and healthcare [32, 33, 72], complemented by targeted searches, we identified 74 DCE studies focusing on healthcare delivery relevant to our context. In total, we identified 33 attributes and classified them into groups (referred to as general concepts; larger dimensions) with similar meanings based on our understanding of the attributes (see document 1 in the electronic supplementary material [ESM]). Eventually, we ended up with eight distinct concepts, whereby several (or no) attributes within one concept potentially may have been selected for the construction of the choice task.

\subsection{Attribute Selection}

\subsubsection{Stakeholders Perspective}

In Switzerland, the importance of timely elicitation of public preferences is strengthened by the influence of direct democracy [10], which allows local populations to be directly involved in decision making (Swiss residents can veto or demand reform through public referenda). For example, in 2012, the Swiss voted on a new plan proposed by the government that aimed to expand managed care nationwide to control cost inflation and address the challenges posed by population aging and chronic diseases [10]. The reforms aimed to improve coordination among networks of healthcare providers, whereby insured patients who joined a managed care network and relinquished their ability to choose their own doctor would benefit from lower costs. However, in the referendum, $76 \%$ of the Swiss public voted against the plan. Therefore, to ensure the acceptability of a newly designed policy, a key step in our DCE development was to involve stakeholders $(N=7)$ (physicians, nurses, insurance and public health representatives, expert patients $[N=2]$, and health authorities) in the development of our DCE right from the initial stage of selecting the most relevant attributes. The expected output of this step was a restricted list of attributes that was relevant to the Swiss context, understandable by and acceptable to the public, and potentially modifiable by policies.
The stakeholder involvement consisted of two parts: an online survey consisting of ranking and rating tasks and a workshop to discuss the final list of attributes. Before the workshop, all stakeholders provided verbal informed consent and signed consent for the session to be recorded. They received online material with key information about the project aims, and the list of eight general concepts with 33 attributes identified in the literature search and with the research expertise of the team. The stakeholders were asked to rank and rate the concepts and attributes from not important to highly important; they were also given the opportunity to suggest other concepts or attributes that were not presented in the list. The outcomes of the online tasks were analyzed and the mean ranks per concept and attribute calculated. At the workshop, the results of the online tasks were presented to the stakeholders, who then exchanged their views on the importance of attributes; consensus was reached in the structured discussion. After the workshop, the final list of attributes with their corresponding levels were sent to the stakeholders for final approval.

\subsubsection{Patients and General Public Perspective}

As we needed to account for the perspectives of the broad target population, we organized three focus groups with six to eight participants aged $\geq 50$ years comprising members of the public and patients and moderated by qualitative researchers. These participants provided written informed consent. Given our time limitations, recruitment challenges, and budgetary constraints, we considered three focus groups to be sufficient, whereby saturation was observed after the third focus group. The goal was to understand how well the participants understood the choice task, whether they could make a trade-off, and what type of scenario presentation (design of the layout) was the easiest for them to perceive visually. At the beginning of each focus group, participants were provided with attributes and their corresponding levels (approved by the stakeholders at a previous stage) and were given time to get familiar with the instructions and definitions. These definitions had been drafted during multiple discussions within the research team, based on the available literature and research practice, and then refined based on feedback from the stakeholders. No prior information was given to participants to avoid influencing their opinions. The participants discussed the relevance and phrasing of the attributes, identified what they considered the most important attributes, and tried making a sample choice task between the two scenarios of care models. We constructed a simple choice task with arbitrary combination of levels to observe the heuristics driving the participants to make tradeoffs, and whether the choice itself was clear and understandable. Second, we wanted to explore participants' perceptions of the conventional DCE format versus using color coding, 
in which different areas (e.g., attributes fixed at the same levels) would be highlighted with color. After each focus group, the research team adapted the phrasing and design according to suggestions from the participants-e.g., related to the attributes' wording, or explanation of terms such as care coordinator, deductibles, and co-payments-to ensure clarity and understandability for the general population. Follow-up was established with focus group participants who expressed interest in the subsequent pilot testing of the online survey.

\subsection{Development of the Survey}

\subsubsection{Survey Structure}

The survey included an introduction that explained its main aims and included demographic questions, an explanation of the DCE task and all the attributes with levels, DCE choice tasks wherein respondents were asked to express which of the presented healthcare scenarios they preferred, and health and healthcare-related questions (see the ESM). The following items were included in the survey:

- self-rated health state [73],

- morbidity status (adapted from the CoLaus Lausanne [74, 75] and Swiss Health Survey 2017 [76]),

- health insurance coverage and healthcare use (from the CoLaus Lausanne survey $[74,75])$,

- health literacy (Health Insurance Literacy [77] and a validated French version of a single screening question assessing Functional Health Literacy [78]), and

- opinions about and trust in the Swiss healthcare system (from the 2011 MOSAICH survey [79]).

\subsubsection{Experimental Design of the Discrete Choice Experiment}

Following a dual-response design [80], the participants were asked first to choose between two unlabeled scenarios and then to repeat their choice with a third option added ("my current model"). This opt-out option was added to the choice task to avoid overestimating the demand for potential new healthcare models.

Fractional factorial design was used in this study, as this facilitates the careful selection of a subset of choice tasks out of all possible combinations [81]. As making choices in healthcare models may be a difficult task for older respondents, we simplified the design of the choice tasks by applying a two-level-overlap constraint: two levels of six were fixed, and the other four levels varied. For each choice task, the algorithm optimized which two attributes to fix at the same levels within the presented scenarios, based on the best resulting efficiency. Such constraint helped improve response efficiency (reducing the task complexity) and the quality of the answers [82, 83]. Reflecting on the estimated overall length of the survey, we decided to give only six experimental choice tasks per respondent. The modified Fedorov algorithm of Ngene software [84] was used to generate an experimental design with 42 choice tasks, which were divided into seven blocks with six tasks each.

In addition to the six experimental choice tasks, each respondent was also asked to complete one practice task and one consistency test. Consistency, the most frequently used approach to assess response quality [85], is measured by comparing respondent choices for the test task and a retest task later in the DCE survey, and counting the number of times the same scenario was chosen. We also measured median time of completion for the entire survey and straight-lining (consistently choosing only the left or right option throughout the survey) to evaluate respondents' engagement and response quality. These measures have been covered by the earlier studies and were considered sufficient for the pilot stage of the survey [85, 86]. Moreover, we also added mouse-over descriptions for response options, allowing respondents to recap the definitions of the attributes. The participants could not skip questions but could return to the previous questions to change their response.

\subsection{Pilot Testing the Survey}

The full survey was integrated in the Qualtrics platform to launch the online pilot testing. An online convenience sample of individuals aged $\geq 50$ years residing in the Frenchspeaking part of Switzerland was used for data collection to obtain the first priors, improve the efficiency of the experimental design, and receive feedback on the layout of the questionnaire. Following standard practice to generate priors, a reasonably small testing sample was considered sufficient. Overall, the pilot aimed to reach 300 completed surveys. The collected choice data were analyzed descriptively to understand the background characteristics of the underlying sample. Then, we used multinomial logit models to model the probability of choosing a given alternative as a function of attribute levels. The estimated regression parameters provided priors to be used for design optimization before the main phase of data collection.

\section{Results}

\subsection{Results from Stakeholder Involvement}

The results of the online task revealed strong agreement among the stakeholders regarding the notions of data 
sharing, care coordination and continuity, and accounting for the needs of the patient. These concepts were considered the most important by all seven stakeholders participating in the project (Table 1). Within the eight concepts, attributes related to data sharing and a person responsible for care were given higher priority (Table 2). Additionally, the inclusion of several important yet subjective characteristics (e.g., level of trust in the care provider, being involved in decision making about provision of care, shared decision making, and patient centeredness) was broadly discussed. However, from the policy perspective, the selected characteristics needed to be measurable and actionable. Therefore, as we were unable to find phrasing that would avoid subjective evaluations of these characteristics, we focused on more measurable ones. Stakeholders suggested several additional attributes, including patient participation, close link with social authorities (social worker available), volunteer inclusion, premium discounts, or allowing all providers covered by the insurance. As a result of the discussions and ideas exchanged at the workshop, which emphasized including informal care and exempting chronic patients from partial payments, the participants of the workshop suggested that we focus on and further develop the eight attributes (Fig. 1). The levels of the attributes were designed based on the existing possibilities within healthcare delivery, practical and scientific knowledge of the research team, and international comparisons. The levels suggested by the research teams were subsequently validated and agreed upon with the stakeholders. The attribute reflecting finance or price (monthly premium change) was included for future calculations of willingness to pay. The levels of the premium changes were based on the average premium in Switzerland and taking a range of 10-20\% increase or decrease, reflecting changes of a reasonable magnitude, while covering a range wide enough to encourage trade-offs.
Table 1 Contingency table for the importance of the eight concepts elicited in the online tasks performed by the stakeholders before the meeting

\begin{tabular}{|c|c|c|c|c|c|}
\hline \multirow[t]{2}{*}{ Concepts } & \multicolumn{5}{|c|}{$\begin{array}{l}\text { Stakeholders who scored the concepts } \\
\text { as follows }(N) \text { : }\end{array}$} \\
\hline & $1^{\mathrm{a}}$ & 2 & 3 & 4 & 5 \\
\hline Data sharing & & & & 2 & 5 \\
\hline Financial characteristics of the health insurance contracts & & 1 & 2 & 1 & 3 \\
\hline Nonfinancial characteristics of the health insurance contracts & & 2 & 2 & 3 & \\
\hline Benefits included in the compulsory basic insurance (LAMaL) & & 1 & 1 & 2 & 3 \\
\hline Choice of healthcare providers & & 1 & & 2 & 4 \\
\hline Quality and efficiency of healthcare providers & & & 1 & 2 & 4 \\
\hline Continuity and coordination of care & & & & 1 & 6 \\
\hline Consideration of patient needs & & & & 1 & 6 \\
\hline
\end{tabular}

a The contents were translated to English from the French original version. The table presents the distribution of the assessments of relative importance as allocated by the stakeholders, where $1=$ not important and $5=$ highly important

LAMaL L'assurance maladie de base obligatoire or obligatory basic health insurance

Table 2 Eight attributes with the highest ranks based on stakeholder responses in the online tasks before the meeting

\begin{tabular}{lll}
\hline Rank & Attribute & Average score $^{\mathrm{a}}$ \\
\hline 1 & Who has access to my patient data? & 4.86 \\
1 & The possibility of a healthcare provider other than the doctor taking charge of certain consultations/aspects of care & 4.86 \\
1 & Patient participation in decision making related to their treatments and care protocols & 4.86 \\
1 & Long-term care & 4.86 \\
5 & Feedback from specialists to the referring general practitioner after a treatment episode (and vice versa) & 4.71 \\
5 & A responsible health professional for the communication and coordination of the various medical services required & 4.71 \\
7 & $\quad$ for treating chronic diseases & 4.64 \\
8 & Person to contact in case of health problems & 4.57 \\
\hline
\end{tabular}

The contents were translated to English from the French original version. The full list of 33 attributes with corresponding scores can be found in document 5 in the electronic supplementary material

${ }^{\mathrm{a}}$ Calculated as the arithmetic mean of attribute ratings, according to the importance scale $1-5$, performed by all seven stakeholders 


\begin{tabular}{|l|l|}
\hline The set of attributes and levels defined after stakeholders' \\
stage and to be tried out with focus groups
\end{tabular}

Fig. 1 The list of attributes and levels after stakeholders' input compared with the final list developed based on feedback from three focus groups. The contents were translated to English from the French

\subsection{Results from Patient and General Public Focus Groups}

The total number of focus group participants was 21, including 11 patients with chronic illness and ten members of the general public (document 2 in the ESM). Based on the focus group discussion, we applied the simplified wording for the descriptions of attributes and levels in the instructions. We based all modifications on wording suggestions from participants as these were more appropriate and understandable for a layperson. Additionally, based on the expressed importance of co-payments for patients with chronic disease, this additional attribute level was included in the list. Second, respondents raised concerns about excessive use of similar constructs, such as care coordination, care navigator, and having healthcare plans

\begin{tabular}{|c|c|}
\hline Attributes & Levels \\
\hline $\begin{array}{l}\text { Which professionals } \\
\text { have access to my } \\
\text { DEP? }\end{array}$ & $\begin{array}{l}\text { - My family doctor only } \\
\text { - All the doctors involved in my care } \\
\text { - All health professionals (doctors and } \\
\text { non-doctors) involved in my care } \\
\text { - All health professionals (doctors and } \\
\text { non-doctors) involved in my care as well } \\
\text { as my health insurance }\end{array}$ \\
\hline $\begin{array}{l}\text { Who coordinates } \\
\text { my care? }\end{array}$ & $\begin{array}{l}\text { - No healthcare professional } \\
\text { - My family doctor } \\
\text { - A health professional who is not a doctor } \\
\text { - A healthcare team including several } \\
\text { healthcare professionals (doctor and } \\
\text { non-doctor) } \\
\text { - A referent (doctor or non-doctor) from } \\
\text { my health insurance }\end{array}$ \\
\hline $\begin{array}{l}\text { Access to the } \\
\text { specialist }\end{array}$ & $\begin{array}{l}\text { - Direct access possible (free choice) } \\
\text { - Need to be referred by a family doctor } \\
\text { (gatekeeping) } \\
\text { - Direct consultation possible if the } \\
\text { specialist is on a list (limited choice) }\end{array}$ \\
\hline $\begin{array}{l}\text { What do insured } \\
\text { with chronic illness } \\
\text { pay? }\end{array}$ & $\begin{array}{l}\text { - Pay both deductible and co-payment } \\
\text { - Pay only co-payment } \\
\text { - Pay only deductible } \\
\text { - Pay neither deductible nor co-payment }\end{array}$ \\
\hline $\begin{array}{l}\text { Formal } \\
\text { compensation for } \\
\text { care and support for } \\
\text { caregivers }\end{array}$ & $\begin{array}{l}\text { - Yes } \\
\text { - Yes, formal compensation and access to } \\
\text { specific services } \\
\text { - No }\end{array}$ \\
\hline $\begin{array}{l}\text { My monthly } \\
\text { premium }\end{array}$ & $\begin{array}{l}\cdot-50 \mathrm{CHF} \\
\cdot-100 \mathrm{CHF} \\
\cdot \text { My monthly premium stays the same } \\
\cdot+50 \mathrm{CHF} \\
\cdot+100 \mathrm{CHF}\end{array}$ \\
\hline
\end{tabular}

original version. $C H F$ Swiss franc, $D E P$ ( Dossier èlectronique du patient) or electronic patient record, GP general practitioner

defined together with health professionals. Therefore, the list of the attributes was shortened (Fig. 1), and an extensive explanation was provided for the attribute of healthcare coordinator, which incorporates the concepts of care navigation and defining care plans (ESM). Regarding the experimental tasks, the respondents found them challenging but relevant, and they could make trade-offs. Additionally, the respondents preferred the design with color-coded areas (e.g., emphasizing attributes fixed at the same level) and descriptions shortened to the key words (Fig. 2).

\subsection{Results from Online Pilot Testing}

\subsubsection{Assessing the Attributes}

We received 305 completed online surveys; we excluded four of these because the completion time was less than 5 
Fig. 2 Example of a discrete choice experiment choice task. The contents were translated to English from the French original version $C H F$ Swiss franc
Which of the following options do you prefer?

\begin{tabular}{|c|c|c|}
\hline & Option 1 & Option 2 \\
\hline $\begin{array}{l}\text { Which professionals have } \\
\text { access to my electronic } \\
\text { patient record? }\end{array}$ & My family doctor only & $\begin{array}{l}\text { All health professionals involved } \\
\text { in my care as well as my health } \\
\text { insurance }\end{array}$ \\
\hline Who coordinates my care? & A healthcare team & No healthcare professional \\
\hline Access to the specialist & $\begin{array}{c}\text { Direct access possible (free } \\
\text { choice) }\end{array}$ & $\begin{array}{l}\text { Direct access possible (free } \\
\text { choice) }\end{array}$ \\
\hline $\begin{array}{l}\text { What do insured with chronic } \\
\text { illness pay? }\end{array}$ & $\begin{array}{c}\text { Pay neither deductible nor co- } \\
\text { payment }\end{array}$ & Pay only co-payment \\
\hline $\begin{array}{l}\text { Formal compensation for care } \\
\text { and support for family } \\
\text { members caregivers }\end{array}$ & Yes & No \\
\hline \multirow[t]{2}{*}{$\begin{array}{l}\text { Variation of my monthly } \\
\text { premium }\end{array}$} & $+50 \mathrm{CHF}$ & $+50 \mathrm{CHF}$ \\
\hline & Option 1 & Option 2 \\
\hline \multirow[t]{2}{*}{ our choice } & $\mathrm{O}_{1}$ & $\otimes_{2}$ \\
\hline & Your current situation & $\begin{array}{c}\text { The option you selected } \\
\text { above }\end{array}$ \\
\hline $\begin{array}{l}\text { you could now keep your } \\
\text { urrent situation: what would } \\
\text { ou choose? }\end{array}$ & $\bigcirc_{1}$ & $\otimes 2$ \\
\hline
\end{tabular}

minutes, which was deemed insufficient to provide quality answers. For the pilot stage, we did not intend to predict the demand for the new healthcare models, thus, the validity of the DCE attributes was assessed by analyzing preference estimates from the forced choices made in the DCE tasks ( $N$ =301). We found that most attributes had signs that matched those from the literature [47, 87] and those expressed during the focus groups. Specifically, attribute levels assumed to yield utility gains (e.g., all doctors having access to the medical file and a family doctor coordinating care) showed the highest positive utility, whereas those yielding potential disutility showed a negative sign (e.g., having a referent person from health insurance coordinating care, or health insurance having access to the medical file). However, the attribute of compensation for informal caregivers turned out to be insignificant, with very small effect sizes (document 3 in the ESM). Nevertheless, we decided to keep this attribute as we expect a lot of heterogeneity and we would be able to reflect on potential preferences in the subgroup analysis. Moreover, the focus groups had indicated that this attribute was very important. Additionally, the monthly premium decrease showed an unexpected negative sign (implying disutility from a premium decrease). We decided not to change the levels of the premium attribute as we covered wide premium variations and we were aware of the potential irregularities that may occur in the pilot phase related to this.

\subsubsection{Assessing the Tasks}

The participants had the opportunity to provide feedback on the complexity and understandability of the tasks. Numerous comments revealed a high level of interest in and the social relevance of the topics raised in the survey: choice, use and costs of health insurance, and views on healthcare in Switzerland overall. In general, the respondents noted the survey's good design and usability, but half the participants mentioned the complexity of the choices. Finally, the quality of the answers was relatively high: median time of completion for the entire survey was 15.5 minutes, $8 \%$ consistently chose only left or right alternatives throughout the survey, and $80 \%$ passed the consistency test (document 4 in the ESM). Additionally, the participants tended to choose their current healthcare model in the follow-up question (chosen in $61 \%$ of choice tasks, and 65 participants consistently chose their current model), as they were not willing to give up the status quo. Moreover, almost half of respondents selfreported a high level of health literacy, and more than $70 \%$ expressed confidence in their choice of health insurance. 


\section{Discussion}

In this article, we have demonstrated our application of a multistage, multi-actor process to develop a DCE survey using a mix of qualitative and quantitative techniques. Such a process ensured that the final DCE design was appropriate and actionable from the perspective of the main stakeholders while remaining understandable and relevant for the target population.

Although the review studies reported an increase in the reporting of DCE development stages [32], it remains limited, likely because of the idea that the process of attribute and level development is less important than other DCE stages [88]. As the current DCE project focuses on a timely healthcare policy issue, involving a lot of interactions between actors and a wide coverage area [11,24], it was crucial to ensure a detailed description of the DCE development stages.

As earlier studies have indicated, there is a lack of rigor in reporting of the attribute development process [69]. Therefore, multiple studies applied different approaches to DCE development because of the specific target population or the complexity and scope of the research question $[19,40,42$, $43,50,89]$. Several earlier studies using DCEs did not provide extensive details on the development process [24, 48, $70,90-92]$. By contrast, our study reports on the application of the mixed-method process of attribute development suggested by the literature review [69] to progress toward a more systematic approach to attribute development. Other studies have used similar approaches to DCE development and reporting, although differences in the survey pilot-testing stage were observed with the small sample performing the think-aloud protocol [20, 47, 66]. In our study, which focused on actionable policy-applied characteristics that could potentially affect the whole population, it was especially important to account for multiple stakeholders and a variety of opinions, which explains our extensive stakeholder engagement and our decision to test the survey online with a larger sample rather than in interviews with a limited number of participants.

In fact, our goal was to ensure that the selected attributes and levels correctly reflected the characteristics of potential healthcare delivery models that were particularly relevant for patients with chronic illness while keeping their number manageable enough so as not to complexify the choice tasks for respondents. We tried to make sure that the multistage process we undertook ensured we did not miss out important characteristics from the patients' perspective. Specifically, we involved two patients who could actively express their needs and opinions in the discussions during the stakeholder stages and all validation processes. On the other hand, we acknowledge that patients' opinions and experiences differ from those of other actors in healthcare and cannot replace the information provided by other stakeholders. At this stage of the DCE development, a greater number of patients might have made it possible to better reflect patients' perspectives and the accuracy of the survey. Consequently, we strived to involve a greater number of patients in the subsequent stages: 11 patients actively participated in the focus groups, and the sample we used for the pilot testing included a large proportion of patients, verifying the acceptability and relevance of the survey. The input from the general public and patients not only helped to improve the design, layout, and instructions but also provided important insights into their valuing of informal care and social solidarity for patients with chronic illness. Moreover, as the DCE attributes, levels, and scenarios should not only be relevant for patients but also actionable for policy makers, we needed to find a consensus among the potentially opposing views of the various stakeholders. Thus, the combination of an online survey and a physical workshop, where all stakeholders were free to express their opinions, followed by extensive involvement of patients in subsequent stages, was considered efficient enough to elicit a large number of ideas while preserving the importance of patients' views.

This DCE development study may be of particular importance to policy makers, health insurance representatives, healthcare managers, and health professionals. The present development study is the first in Switzerland to focus on the preferences of multiple actors regarding healthcare delivery and the value of coordinated care models. Additionally, it contributes to the body of literature on DCEs, advancing the use of more detailed and standardized approaches to the reporting of DCE development. Finally, our study emphasizes the importance of involving various stakeholders, as the diversity of views helped to reflect a real-world picture and ensure policy relevance. Moreover, this emphasizes the importance for similar future research, as the involvement of various stakeholders from the early stage is likely to promote the dissemination of results and increase credibility and acceptance within the population. We are aware that the healthcare delivery system is a complex construct with many aspects to consider, and we acknowledge the limitations of our study related to the DCE format and associated restrictions of the number of final attributes included. As such, we also acknowledge that we were unable to present all possible important characteristics of the complex healthcare delivery system in the DCE choice task, as more than six to eight attributes would be difficult for participants to comprehend. Accordingly, the majority of studies investigating more specific healthcare research areas used designs with five to eight attributes [24, 37, 39, 42, 43, 47, 48, 52, 70]. By contrast, several studies had designs with 10 or 11 attributes; these authors acknowledged this as a limitation for cognitive processing [50], used partial profiles to reduce 
the number of attributes [89], or used a single-scenario presentation format, asking the participants whether or not they would accept such a scenario [19], which is very different from our approach. Second, in terms of policy making we focused only on actionable characteristics within the healthcare system. Therefore, potentially important aspects such as patient centeredness or shared decision making were deemed unfeasible to express in an impersonal manner, not modified by individual bias. Third, although appropriate for the pilot-testing stages of DCEs, the results of the pilot testing were derived from a small sample. The future main study will involve a large sample of 1000 respondents required to elicit public preferences from French-speaking parts of Switzerland. Moreover, we will be able to analyze whether participants are willing to make trade-offs, the types of trade-offs they are willing to make (e.g., give up freedom of provider choice or pay higher premiums for the benefits of coordinated care or valorization of informal care, etc.), and the monetary values of such trade-offs. Additionally, including questions on health status, healthcare use, and opinions about the healthcare system in Switzerland will allow us to perform a subgroup analysis to explore heterogeneity and provide more insights that would be valuable from a policy perspective. Finally, since potential care models will likely focus on care coordination, one may expect to consider several attributes specific to care coordination, whereas we used only one. This decision presumably limits the risk of ambiguity, as care coordination can be expressed in multiple ways and there is no universal definition for it. Therefore, expressing care coordination in multiple attributes was likely to cause confusion among the respondents, who would judge them as interconnected, resulting in redundancy or illogical combinations, which was discussed in the focus groups.

\section{Conclusions}

This article will be useful to researchers designing DCEs and who are willing to inform the attribute selection and design validation stages for a broad health policy perspective. Such a study would be of special interest in Switzerland, where preferences on organizational aspects of the healthcare system and access to care are rarely elicited. In our study, a multistage, mixed-methods approach involving multiple actors was essential for the identification and selection of attributes important for policy makers and for the general public and patients. Our use of mixed methods allowed us to account for and align various perspectives. Moreover, various stages provided important feedback on the redundancy of attributes with similar meanings related to care coordination and meant we could focus more on informal care and financial barriers for patients with chronic illnesses.
Supplementary Information The online version contains supplementary material available at https://doi.org/10.1007/s40271-021-00568-2.

\section{Declarations}

Funding This study was funded by the Swiss National Science Foundation (SNSF) within Smarter health care-National Research Programme (NRP 74) and Grant number (407440_183447).

Conflicts of interest Anna Nicolet, Clémence Perraudin, Joël Wagner, Ingrid Gilles, Nicolas Krucien, Isabelle Peytremann-Bridevaux, and Joachim Marti have no conflicts of interest that are directly relevant to the content of this article.

Availability of data and material The model and participants' characteristics from the pilot testing are provided in the ESM. The full dataset is available from the authors on request.

Ethics approval As this study did not concern a human disease or the structure and functioning of the human body, the Cantonal Commission for the Ethics of Research on Human Beings (CER-VD, Lausanne, Switzerland) waived ethics approval for this study (protocol submitted, Req-2019-01265) as it did not fall within the scope of the research involving human beings and did not require authorization from the ethics committee to be carried out.

Consent Not applicable.

Author contributions JM obtained funding, designed the concept of the study, supervised the analysis and interpretation of the results, and revised the manuscript. AN was responsible for data preparation, primary analysis, and manuscript preparation. $\mathrm{CP}$ was responsible for participant recruitment and communication. IG organized and moderated the qualitative sessions. NK provided statistical support and DCE expertise. All authors contributed to the study design and development, data analysis, and revision of the manuscript.

Open Access This article is licensed under a Creative Commons Attribution-NonCommercial 4.0 International License, which permits any non-commercial use, sharing, adaptation, distribution and reproduction in any medium or format, as long as you give appropriate credit to the original author(s) and the source, provide a link to the Creative Commons licence, and indicate if changes were made. The images or other third party material in this article are included in the article's Creative Commons licence, unless indicated otherwise in a credit line to the material. If material is not included in the article's Creative Commons licence and your intended use is not permitted by statutory regulation or exceeds the permitted use, you will need to obtain permission directly from the copyright holder. To view a copy of this licence, visit http://creativecommons.org/licenses/by-nc/4.0/.

\section{References}

1. Prince MJ, et al. The burden of disease in older people and implications for health policy and practice. Lancet. 2015;385(9967):549-62.

2. Lawless MT, et al. What does integrated care mean from an older person's perspective? A scoping review. Bmj Open. 2020;10:e035157. 
3. van der Heide I, et al. Patient-centeredness of integrated care programs for people with multimorbidity. Results from the European ICARE4EU project. Health Policy. 2018;122(1):36-43.

4. Valentijn PP, et al. Understanding integrated care: a comprehensive conceptual framework based on the integrative functions of primary care. Int J Integr Care. 2013;13:e010.

5. Grone O, Garcia-Barbero M, W.H.O.E.O.f.I.H.C. Services. Integrated care: a position paper of the WHO European Office for Integrated Health Care Services. Int J Integr Care. 2001;1:e21.

6. Droz M, Senn N, Cohidon C. Communication, continuity and coordination of care are the most important patients' values for family medicine in a fee-for-services health system. BMC Fam Pract. 2019;20(1):19.

7. Espinosa-Gonzalez $\mathrm{AB}$, et al. The role of the state in financing and regulating primary care in Europe: a taxonomy. Health Policy. 2021;125(2):168-76.

8. Focus on spending in primary care:first estimates. OECD Policy Brief, 2018. https://www.oecd.org/health/health-systems/Spend ing-on-Primary-Care-Policy-Brief-December-2018.pdf.

9. Schussele Filliettaz S, et al. Integrated care in Switzerland: Results from the first nationwide survey. Health Policy. 2018;122(6):568-76.

10. De Pietro C, et al. Switzerland: health system review. Health Syst Transit. 2015;17(4):1-288 (xix).

11. Reich O, Rapold R, Flatscher-Thoni M. An empirical investigation of the efficiency effects of integrated care models in Switzerland. Int J Integr Care. 2012;12:e2.

12. Tsiachristas A, et al. Performing economic evaluation of integrated care: highway to hell or stairway to heaven? Int J Integr Care. 2016;16(4):1-12.

13. Cohidon C, Cornuz J, Senn N. Primary care in Switzerland: evolution of physicians' profile and activities in twenty years (19932012). BMC Fam Pract. 2015;16:107.

14. Katz EB, et al. Comparative effectiveness of care coordination interventions in the emergency department: a systematic review. Ann Emerg Med. 2012;60(1):12-23e1.

15. Gorin SS, et al. Cancer care coordination: a systematic review and meta-analysis of over 30 years of empirical studies. Ann Behav Med. 2017;51(4):532-46.

16. Gaebel, W., et al., EPA guidance on the quality of mental health services: A systematic meta-review and update of recommendations focusing on care coordination. European Psychiatry, 2020. $63(1)$.

17. Reeves E, Schweighoffer R, Liebig B. An investigation of the challenges to coordination at the interface of primary and specialized palliative care services in Switzerland: a qualitative interview study. J Interprof Care. 2021;35(1):21-7.

18. Schultz EM, McDonald KM. What is care coordination? Int J Care Coord. 2014;17(1-2):5-24

19. Krucien N, Le Vaillant M, Pelletier-Fleury N. What are the patients' preferences for the Chronic Care Model? An application to the obstructive sleep apnoea syndrome. Health Expect. 2015;18(6):2536-48.

20. Muhlbacher AC, et al. Patient preferences for features of health care delivery systems: a discrete choice experiment. Health Serv Res. 2016;51(2):704-27.

21. Jasper R, et al. Practitioners preferences of care coordination for older people: a discrete choice experiment. J Gerontol Soc Work. 2018;61(2):151-70.

22. Karam M, et al. Nursing care coordination for patients with complex needs in primary healthcare: a scoping review. Int $\mathbf{J}$ Integr Care. 2021;21(1).

23. Meeker D, et al. Use of Insurance Against a Small Loss as an Incentive Strategy. Decis Anal. 2015;12(3):122-9.
24. Zweifel P. Swiss experiment shows physicians, consumers want significant compensation to embrace coordinated care. Health Aff. 2011;30(3):510-8.

25. Muhlbacher A, Johnson FR. Choice experiments to quantify preferences for health and healthcare: state of the practice. Appl Health Econ Health Policy. 2016;14(3):253-66.

26. Denburg AE, et al. Does moral reasoning influence public values for health care priority setting?: A population-based randomized stated preference survey. Health Policy. 2020;124(6):647-58.

27. Schneider F, Buser T. Promising degrees of stakeholder interaction in research for sustainable development. Sustain Sci. 2018;13(1):129-42.

28. Green C, Gerard K. Exploring the social value of health-care interventions: a stated preference discrete choice experiment. Health Econ. 2009;18(8):951-76.

29. Mangin D, et al. Making patient values visible in healthcare: a systematic review of tools to assess patient treatment priorities and preferences in the context of multimorbidity. BMJ Open. 2016;6(6): 010903 .

30. Muhlbacher AC. Patient-centric HTA: different strokes for different folks. Expert Rev Pharmacoecon Outcomes Res. 2015;15(4):591-7.

31. Harvey G, et al. Achieving integrated care for older people: shuffling the deckchairs or making the system watertight for the future? Int J Health Policy Manag. 2018;7(4):290-3.

32. de Bekker-Grob EW, Ryan M, Gerard K. Discrete choice experiments in health economics: a review of the literature. Health Econ. 2012;21(2):145-72.

33. Clark MD, et al. Discrete choice experiments in health economics: a review of the literature. Pharmacoeconomics. 2014;32(9):883-902.

34. Stolk EA, et al. Discrete choice modeling for the quantification of health states: the case of the EQ-5D. Value Health. 2010;13(8):1005-13.

35. Nicolet A, Groothuis-Oudshoorn CGM, Krabbe PFM. Does inclusion of interactions result in higher precision of estimated health state values? Value in Health. 2018;21(12):1437-44.

36. Turner D, et al. Do patients value continuity of care in general practice? An investigation using stated preference discrete choice experiments. J Health Serv Res Policy. 2007;12(3):132-7.

37. Richardson $\mathrm{G}$, et al. What outcomes are important to patients with long term conditions? A discrete choice experiment. Value Health. 2009;12(2):331-9.

38. Negrín MA, Pinilla J, León CJ. Willingness to pay for alternative policies for patients with Alzheimer's disease. Health Econ Policy Law. 2008;3(3):257-75.

39. Akkazieva B, et al. Patients' preferences for healthcare system reforms in Hungary. Appl Health Econ Health Policy. 2006;5(3):189-98.

40. Burton $\mathrm{CD}$, et al. The value of different aspects of person-centred care: a series of discrete choice experiments in people with longterm conditions. BMJ Open. 2017;7(4):e015689.

41. Gerard K, et al. The introduction of integrated out-of-hours arrangements in England: a discrete choice experiment of public preferences for alternative models of care. Health Expect. 2006;9(1):60-9.

42. Murchie P, et al. Determining cancer survivors' preferences to inform new models of follow-up care. Br J Cancer. 2016;115(12):1495-503.

43. Lagarde M, Erens B, Mays N. Determinants of the choice of GP practice registration in England: evidence from a discrete choice experiment. Health Policy. 2015;119(4):427-36.

44. Dixon S, et al. Assessing patient preferences for the delivery of different community-based models of care using a discrete choice experiment. Health Expect. 2015;18(5):1204-14. 
45. Wellman GS, Vidican C. Pilot study of a hierarchical Bayes method for utility estimation in a choice-based conjoint analysis of prescription benefit plans including medication therapy management services. Res Social Adm Pharm. 2008;4(3):218-30.

46. Wittink MN, et al. Towards patient-centered care for depression. The Patient. 2010;3(3):145-57.

47. Determann D, et al. What health plans do people prefer? The trade-off between premium and provider choice. Soc Sci Med. 2016;165:10-8.

48. Struik MHL, et al. Preference Structure of Clinicians in the Use of Electronic Medical Records; Quantifying the Relative Importance of Barriers and Facilitators of an Innovation. Value in Health. 2013;16(7):A471.

49. Huppelschoten AG, et al. The monetary value of patient-centred care: results from a discrete choice experiment in Dutch fertility care. Hum Reprod. 2014;29(8):1712-20.

50. Groenewoud S, et al. What influences patients' decisions when choosing a health care provider? Measuring preferences of patients with knee arthrosis, chronic depression, or Alzheimer's disease, using discrete choice experiments. Health Serv Res. 2015;50(6):1941-72.

51. Bessen T, et al. What sort of follow-up services would Australian breast cancer survivors prefer if we could no longer offer long-term specialist-based care? A discrete choice experiment. Br J Cancer. 2014;110(4):859-67.

52. Whitty JA, et al. Patient preferences and willingness-to-pay for a home or clinic based program of chronic heart failure management: findings from the Which? trial. PLoS ONE. 2013;8(3):e58347.

53. Oliver D, et al. Patient trade-offs between continuity and access in primary care interprofessional teaching clinics in Canada: a cross-sectional survey using discrete choice experiment. BMJ Open. 2019;9(3):e023578.

54. Abiiro GA, et al. Developing attributes and attribute-levels for a discrete choice experiment on micro health insurance in rural Malawi. Bmc Health Serv Res. 2014;14:1-5.

55. Ozawa S, Grewal S, Bridges JF. Household size and the decision to purchase health insurance in cambodia: results of a discretechoice experiment with scale adjustment. Appl Health Econ Health Policy. 2016;14(2):195-204.

56. Obse A, et al. Eliciting preferences for social health insurance in Ethiopia: a discrete choice experiment. Health Policy Plan. 2016;31(10):1423-32.

57. Larson E, et al. Moving toward patient-centered care in africa: a discrete choice experiment of preferences for delivery care among 3,003 Tanzanian women. PLoS ONE. 2015;10(8): 00135621.

58. Hanson K, et al. Preferences for hospital quality in Zambia: results from a discrete choice experiment. Health Econ. 2005;14(7):687-701.

59. Coast J, Horrocks S. Developing attributes and levels for discrete choice experiments using qualitative methods. J Health Serv Res Policy. 2007;12(1):25-30.

60. Coast J, et al. Using qualitative methods for attribute development for discrete choice experiments: issues and recommendations. Health Econ. 2012;21(6):730-41.

61. Lancsar E, Louviere J. Conducting discrete choice experiments to inform Healthcare decision making. Pharmacoeconomics. 2008;26(8):661-77.

62. Klojgaard ME, Bech M, Sogaard R. Designing a stated choice experiment: the value of a qualitative process. J Choice Model. 2012;5(2):1-18.

63. Bridges JFP, et al. Conjoint analysis applications in healtha checklist: a Report of the ISPOR Good Research Practices for Conjoint Analysis Task Force. Value in Health. 2011;14(4):403-13.
64. Janssen EM, Segal JB, Bridges JFP. A framework for instrument development of a choice experiment: an application to type 2 diabetes. Patient-Patient Cent Outcomes Res. 2016;9(5):465-79.

65. Obadha M, et al. Attribute development and level selection for a discrete choice experiment to elicit the preferences of health care providers for capitation payment mechanism in Kenya. Health Econ Rev. 2019;9(1):1-19.

66. Barber S, et al. Development of a discrete-choice experiment (DCE) to elicit adolescent and parent preferences for hypodontia treatment. Patient-Patient Cent Outcomes Res. 2019;12(1):137-48.

67. Vass C, Rigby D, Payne K. The role of qualitative research methods in discrete choice experiments: a systematic review and survey of authors. Med Decis Making. 2017;37(3):298-313.

68. Chudner I, et al. Video or in-clinic consultation? Selection of attributes as preparation for a discrete choice experiment among key stakeholders. Patient-Patient Cent Outcomes Res. 2019;12(1):69-82.

69. Helter TM, Boehler CEH. Developing attributes for discrete choice experiments in health: a systematic literature review and case study of alcohol misuse interventions. J Substance Use. 2016;21(6):662-8.

70. Becker K, Zweifel P. Age and choice in health insurance evidence from a discrete choice experiment. Patient-Patient Cent Outcomes Res. 2008;1(1):27-40.

71. Gesundheitsobservatorium S. Gesundheit in der Schweiz Fokus chronische Erkrankungen Nationaler Gesundheitsbericht. Bern: Hogrefe Verlag; 2015.

72. Soekhai V, et al. Discrete choice experiments in health economics: past present and future. Pharmacoeconomics. 2019;37(2):201-26.

73. Eriksson I, Unden AL, Elofsson S. Self-rated health. Comparisons between three different measures. Results from a population study. Int J Epidemiol. 2001;30(2):326-33.

74. Kraege V, et al. Validation of seven type 2 diabetes mellitus risk scores in a population-based cohort: the CoLaus Study. J Clin Endocrinol Metab. 2020;105(3):E265-72.

75. Firmann M, et al. The CoLaus study: a population-based study to investigate the epidemiology and genetic determinants of cardiovascular risk factors and metabolic syndrome. Bmc Cardiovasc Disord. 2008;8:1-11.

76. Bundesamt für Statistik B. Die Schweizerische Gesundheitsbefragung 2017 in Kürze. Konzept, Methode, Durchführung. 2018.

77. Paez KA, et al. Development of the health insurance literacy measure (HILM): conceptualizing and measuring consumer ability to choose and use private health insurance. J Health Commun. 2014;19:225-39.

78. Zuercher E, et al. Health literacy and quality of care of patients with diabetes: a cross-sectional analysis. Prim Care Diabetes. 2017;11(3):233-40.

79. Vandenplas $\mathrm{C}$, et al. Identifying pertinent variables for nonresponse follow-up surveys: lessons learned from four cases in Switzerland. Survey Research Methods. 2015;9(3):141-58.

80. Brazell JD, et al. The no-choice option and dual response choice designs. Mark Lett. 2006;17(4):255-68.

81. Box GE, Hunter JS, Hunter WG. Statistics for experimenters: design, innovation, and discovery. 2nd ed.: Wiley, 2005.

82. Jonker MF, et al. Effect of Level Overlap and Color Coding on Attribute Non-Attendance in Discrete Choice Experiments. Value Health. 2018;21(7):767-71.

83. Jonker MF, et al. Attribute level overlap (and color coding) can reduce task complexity, improve choice consistency, and decrease the dropout rate in discrete choice experiments. Health Econ. 2019;28(3):350-63. 
84. ChoiceMetrics. 1.2 User Manual \& Reference Guide. ChoiceMetrics Pty Ltd.: Sydney,Australia. 2018. http://www.choicemetrics.com/NgeneManual120.pdf.

85. Pearce A, et al. Respondent understanding in discrete choice experiments: a scoping review. Patient. 2021;14(1):17-53.

86. Johnson FR, Yang JC, Reed SD. The internal validity of discrete choice experiment data: a testing tool for quantitative assessments. Value Health. 2019;22(2):157-60.

87. Determann D, et al. Personal health records in the Netherlands: potential user preferences quantified by a discrete choice experiment. J Am Med Inform Assoc. 2017;24(3):529-36.

88. De Brun A, et al. A novel design process for selection of attributes for inclusion in discrete choice experiments: case study exploring variation in clinical decision-making about thrombolysis in the treatment of acute ischaemic stroke. BMC Health Serv Res. 2018;18(1):483.
89. Kessels R, et al. How to reform western care payment systems according to physicians, policy makers, healthcare executives and researchers: a discrete choice experiment. BMC Health Serv Res. 2015;15:191.

90. Whitty JA, Scuffham PA, Rundle-Thielee SR. Public and decision maker stated preferences for pharmaceutical subsidy decisions. Appl Health Econ Health Policy. 2011;9(2):73-9.

91. van der Wulp I, et al. Societal preferences for standard health insurance coverage in the Netherlands: a cross-sectional study. BMJ Open. 2012;2(2):e001021.

92. Rischatsch M, Zweifel P. What do physicians dislike about managed care? Evidence from a choice experiment. Eur J Health Econ. 2013;14(4):601-13. 